\title{
Dichorionic Twin Pregnancy Discordant for Fetal Anencephaly: A Case Report
}

\author{
Mohamed Mutasim Mohamed Ahamed ${ }^{1 *}$, Alla Mohamed Ali Shakout ${ }^{1}$ and Siddig Omer M Handady ${ }^{2}$ \\ ${ }^{1}$ Specialist of Obstetrics and Gynecology, Reproductive Health Care Centre, Sudan \\ ${ }^{2}$ Department of Obstetrical \& Gynecology, Ibrahim Malik hospital, Sudan
}

*Corresponding author: Mohamed Mutasim Mohamed Ahamed, Specialist of Obstetrics and Gynecology, Reproductive Health Care Centre, Khartoum, Sudan.

Received Date: December 28, 2019

Published Date: March 15, 2019

\begin{abstract}
Background: Anencephaly is a lethal diagnosis. In twin pregnancies discordant for anencephaly, there are risks of development of polyhydramnios, severe preterm delivery and death of an encephalic fetus.

Case presentation: A 30-year-old G 111 Para 11 both were vaginal delivery presented at Ian Donald ultrasound department for first time scan at 27-week gestational age. Ultrasound showed a live dichorionic, diamniotic intrauterine twin pregnancy. The Gestational age was 27 weeks and 5 days. A cranium of twin A was described. Polyhydramnios was noted for twin B at 29+5 weeks. After adequate obstetric, genetic counseling and given all the information about the risks involving this pregnancy, the parents opted for conservative management without any intervention.
\end{abstract}

Conclusion: The early diagnosis of anencephaly before 16 weeks' gestational age in the twin decrease morbidity and mortality to the unaffected normal twin by providing the option of selective fetal reduction at an optimal gestational age.

Keywords: Anencephaly; Twin pregnancy; Discordant fetal growth; Ultrasound

\section{Introduction}

Anencephaly is a lethal diagnosis. In twin pregnancies discordant for anencephaly, there are risks of development of polyhydramnios, severe preterm delivery and death of the anencephalic fetus [1].

The widespread introduction of ultrasound screening at $11+0$ to $13+6$ weeks of gestation allowed an earlier diagnosis of major fetal anomalies such as anencephaly [2-4]. Twin pregnancies present with a higher prevalence of anencephaly, with monochorionic twins having a higher incidence of discordance than dichorionic [5]. Twin pregnancies resulting from assisted reproductive treatments (ART) are particularly affected by this condition [6]. In dichorionic twins discordant for anencephaly, there are three management options: selective foeticide, serial ultrasound examination for polyhydramnios or expectant management [1]. In this case, the authors report a case of twin pregnancy discordant for anencephaly which was managed conservatively.

\section{Case Report}

A 30-year-old G 111 Para 11 both were vaginal delivery presented at Ian Donald ultrasound department for first time at 27 weeks. Ultrasound showed a live dichorionic, diamniotic intrauterine twin pregnancy. The Gestational age was 27 weeks and 5 days Acrania of twin A was described, based on the absence of the cranial vault and cerebral hemispheres. Polyhydramnios was noted for twin B at 29+5 weeks. At 29 weeks and 5 days both twins ware cephalic with estimated fetal weight 1.5 kilogram for twin $\mathrm{A}$ And 1.2 kilogram for twin B. After adequate obstetric and genetic counseling and given all the information about the risks involving this pregnancy, the parents opted for conservative management without any intervention.

One week later the patient presented with advance preterm labor and delivered the twins vaginaly.

At birth, twin A weighed 1.5 kilograms, with a normal physical exam. Twin B was anencephalic and weighed 1.2 kilograms. Apgar scores for twin A were 9 and 9 at 1 and 5 minutes. Apgar scores for twin B were 4 and 4 at 1 and 5 minutes.

Twin B expired 2 hours after birth. Autopsy and genetic analysis of twin B were declined. Twin A was dead after 7 days at hospital due to sepsis (Figures 1-3). 

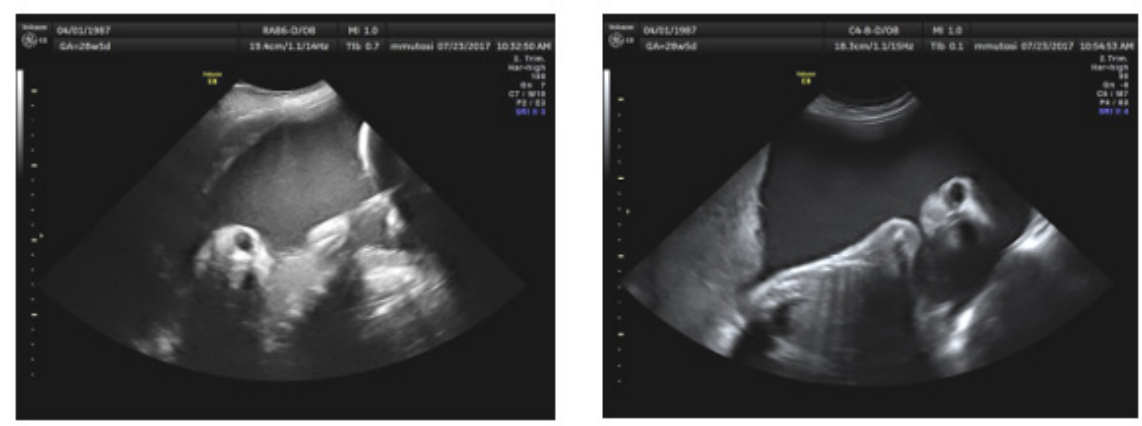

Figure 1: US of anencephalic fetus.

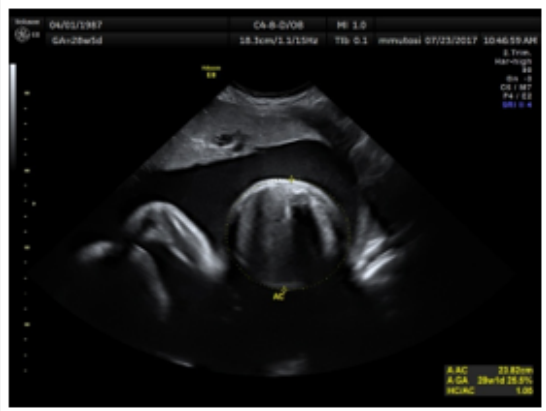

Figure 2a AC of normal fetus

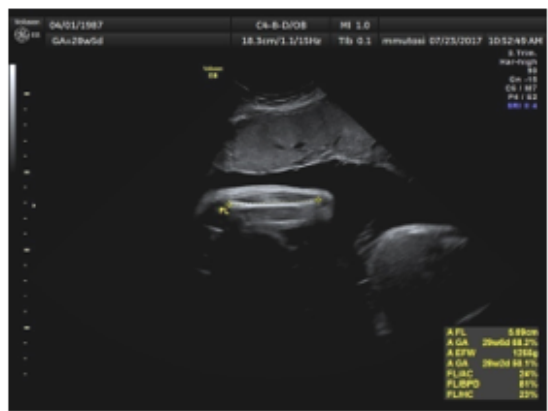

Figure 2b FL of normal fetus

Figure 2
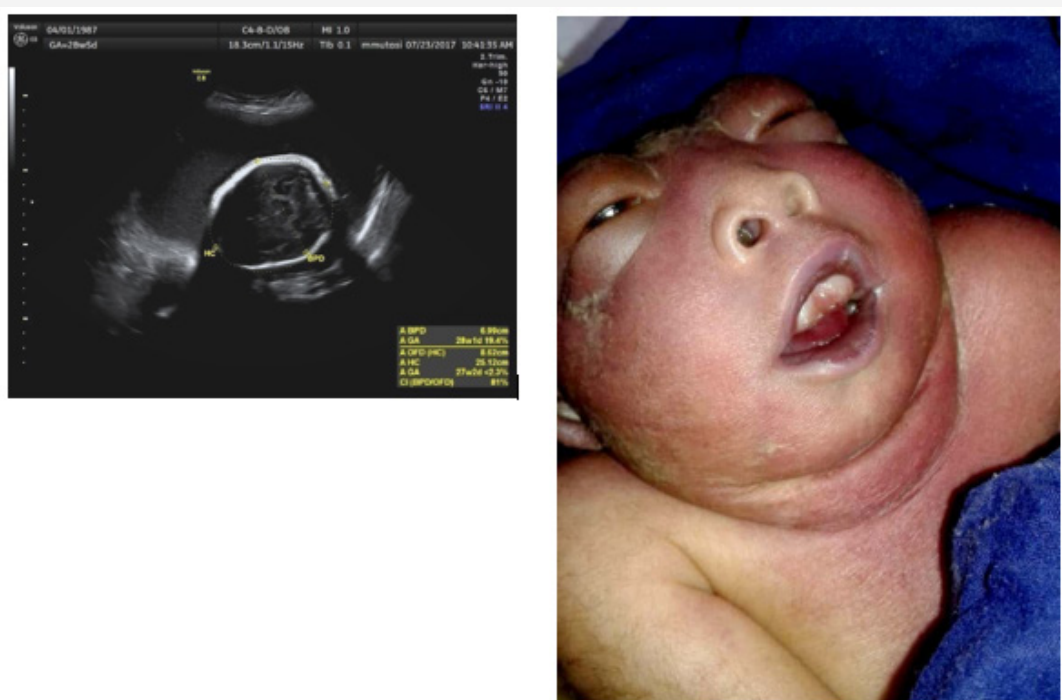

Figure 3: $\mathrm{HC}$ and BPD of normal fetus.

\section{Discussion}

Studies have reported that, among twin pregnancies, neural tube defects, such as anencephaly, are more common when compared with singletons [7]. But causes still not identified, and it was assumed to be attributed to the twinning or to the mode of conception.

But in the current case report, there is a delay in detecting the abnormality which was observed in 27 weeks of gestational age due to patients delay. The absence of the cranial vault and cerebral hemispheres, hallmarks of anencephaly, can theoretically be distinguished as early as at 9 weeks' gestation, when the skeletogenous layer around the brain begins to develop [8]. Anencephaly diagnosis can be obviously suggested at the routine sonogram during tenth to 14 th week of GA in singleton pregnancies, with previous consideration in mind for such findings [9]. Late progress in ultrasound diagnoses enabled accessibility to observe the preserved mid-brain, brainstem, and facial structures, while in early cases of anencephaly, the only finding may be acrania.

Management options are selective foetocide of the abnormal twin, expectant management by continuing pregnancy without intervention [10] or amnioreduction of polyhydramnios. In the 
present case report, the patients refused the decision of selective foetocide, and accordingly expectant management is the second option, while labor pain prevented applying amnioreduction.

Selective termination of the anomalous fetus with dichorionic placentation is a safe and effective option in expert hands, although there is a risk of miscarriage or preterm delivery of the normal cotwin. Because of these risks, expectant management may be a safer option if the twin with the anomaly is not expected to have prolonged survival or a favorable outcome (eg, trisomy 18) [11].

\section{Conclusion}

The early diagnosis of anencephaly before 16 weeks' gestational age in the twin decrease morbidity and mortality to the unaffected normal twin by providing the option of selective fetal reduction at an optimal gestational age. Polyhydramnios is a common complication of anencephalic gestations discordant for anencephaly serial ultrasound examinations may help minimize the risk of miscarriage by guiding therapeutic amnioreduction. periconceptional planning for folic acid.

\section{Acknowledgement}

I acknowledge the cooperation of Ian Donald ultrasound department and EL-academy Maternity Hospital residents who participated in appointing the patient and following up. We also appreciate the commitment and compliance of the patient who reported the required data and attended for the regular follow up.

\section{Conflict of Interest}

No conflict of interest.

\section{References}

1. Leeker M, Beinder E (2004) Twin pregnancies discordant for anencephaly-management, pregnancy outcome and review of literature. Eur J Obstet Gynecol Reprod Biol 114(1): 15-8.

2. Batista J, Raposo L, Sousa A, Cerveira I (2013) Discordance for fetal anencephaly in a dichorionic twin pregnancy: A case report. Open Journal of Obstetrics and Gynecology 3(7): 541-543.

3. Souka AP, Nicolaides KH (1997) Diagnosis of fetal abnormalities at the 10-14 weeks scan. Ultrasound Obstet Gynecol 10(6): 429-442.

4. Jonhson S, Sebire N, Snijders R, Tunkel S, Nicolaides K (1997) Ultrasound screening for anencephaly at 10 - 14 weeks of gestation. Ultrasound Obstet Gynecol 9(1): 14-16.

5. Sebire NJ, Sepulveda W, Hughes KS, Noble P, Nicolaides KH (1997) Management of twin pregnancies discordant for anencephaly. Br J Obstet Gynaecol 104(2): 216-219.

6. Ben-Ami I, Edel Y, Barel O, Vaknin Z, Herman A, et al. (2011) Do assisted conception twins have an increased risk for anencephaly? Hum Reprod 26(12): 3466-3471.

7. Ben-Ami I, Vaknin Z, Reish O, Sherman D, Herman A, et al. (2005) Is there an increased rate of anencephaly in twins?" Prenat Diagn 25(11): $1007-$ 1010 .

8. Blaas HG, Eik-Nes SH (2009) Sonoembryology and early prenatal diagnosis of neural anomalies. Prenat Diagn 29(4): 312-325.

9. Taşcı Y, Karasu Y, Erten O, Karadağ B, Göktolga U (2012) Dichorionic twin pregnancy discordant for fetal anencephaly: a case report. J Turk Ger Gynecol Assoc 13(1): 64-66.

10. Chasen ST, Chervenak FA (2017) Twin pregnancy: Prenatal issues.

11. Rustico MA, Baietti MG, Coviello D, Orlandi E, Nicolini U (2005) Managing twins discordant for fetal anomaly. Prenat Diagn 25(9): 766-771. 